\title{
Consensus guidelines for the investigation and management of encephalitis
}

\author{
A summary of a position paper for Australian and New Zealand practitioners
}

-

ncephalitis is caused by inflammation of the brain and is a challenging condition for clinicians to identify and manage. It manifests as a complex neurological syndrome with protean clinical manifestations that may be caused by a large number of aetiologies, many without effective treatments. It can be fatal and survivors often experience significant neurological morbidity. Studies have shown variable quality in case management in multiple settings, ${ }^{1-3}$ emphasising the need for consensus guidelines.

The need for guidelines is also important because encephalitis is a marker of emerging and re-emerging infectious diseases, and is therefore a syndrome of public health importance. There are unique infectious aetiologies in Australia - including Hendra virus, Australian bat lyssavirus, Murray Valley encephalitis virus and West Nile virus (Kunjin virus) infections - that require early identification, reporting and specialist clinical and public health responses. Regionally, causes of encephalitis with potential for introduction into and epidemic activity in Australia include Japanese encephalitis virus, enterovirus 71, dengue virus and Nipah virus. There is also a rapidly growing list of immune-mediated encephalitides that are important because of their potential response to immunomodulatory treatments and their association with underlying tumours.

\section{"encephalitis is a marker of emerging and re-emerging infectious diseases"}

Keith Eastwood DrPH, MAppEpi, BAppSci

Bruce J Brew $M B B S, M D, F R A C P^{4}$

Yusuf Nagree MBBS, FACEM, MBA(E) $)^{5,6}$

Cheryl A Jones MBBS(Hons), PhD, FRACP,

1 University of Sydney, Sydney, NSW.

2 The Children's Hospital at Westmead Sydney, NSW.

3 Hunter New England Health, Newcastle, NSW.

4 St Vincent's Hospital, Sydney, NSW.

5 Fremantle Hospital, Fremantle, WA 6 University of Western Australia, Fremantle, WA

philip.britton@ health.nsw.gov.au

Although comprehensive guidelines have been published elsewhere, including recent international consensus guidelines, ${ }^{4}$ these are detailed and lack a specific geographic focus. As a result, we have developed a concise guideline for clinicians in Australia and New Zealand (doi: 10.1111/imj.12749) ${ }^{5}$ that provides a substantial update to previous guidance published in the Journal. ${ }^{6}$

The guideline was developed by the Australasian Society for Infectious Diseases Clinical Research Network (ASID CRN) Encephalitis Special Interest Group with subsequent, multiple rounds of consultation involving the ASID Guidelines Committee, the Public Health Association of Australia, the Australian and New Zealand Association of Neurologists and the Australasian College for Emergency Medicine.

\section{Main recommendations}

The guideline principally consists of two algorithms. The first algorithm addresses the patient with possible meningoencephalitis - a scenario that is frequently

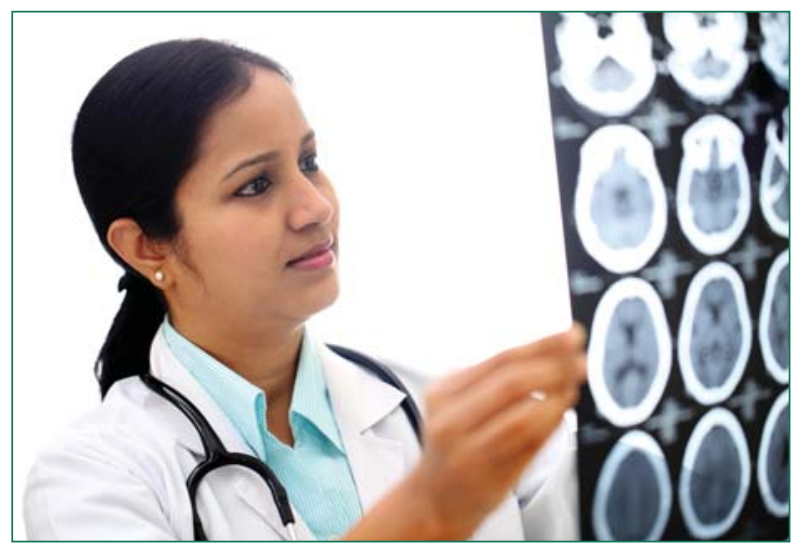

encountered in emergency departments. This algorithm is designed to assist clinicians to: consider encephalitis within a wide differential diagnosis, perform appropriate specimen sampling and investigations, and initiate antimicrobial therapy promptly (including acyclovir for possible herpes simplex virus [HSV] encephalitis). In most patients with possible meningoencephalitis, an alternative diagnosis will be made. The algorithm aims to discriminate between patients in whom encephalitis can be excluded and those who require a more detailed assessment. The second algorithm addresses the patient in whom encephalitis is considered likely. This algorithm provides a robust clinical case definition of encephalitis, identifies key first-line (universal) diagnostic tests (Box), outlines a process of excluding HSV disease, and formulates an approach of directed (second- and third-line) diagnostic testing based on risk factors, clinical features and radiological features.

In addressing these scenarios, the guideline answers the following questions:

- What features are important to consider during history-taking and examination?

- In which patients should magnetic resonance imaging be performed?

- What are the common abnormalities evident in cerebrospinal fluid?

Furthermore, it provides advice on the tests that should be done to diagnose the most common causes of encephalitis and defines specific patient subpopulations to highlight differences in aetiology (to help prioritise testing). These subpopulations include: children and neonates, immunocompromised patients, overseas travellers and immigrants, and patients residing in tropical Australia.

The guideline considers the particularly vexing question of the contemporary role of brain biopsy by presenting 
Recommended first-line investigation of encephalitis in Australia and New Zealand*5

Specimen/Investigation

$\mathrm{CSF} \dagger$

Serumll

Respiratory

Faeces

Skin swabs (where

lesions present)

Neuroimaging

EEG

†Collect up to $10 \mathrm{~mL}$, if able, in four tubes in adults and children, and up to $5 \mathrm{~mL}$ in a small child ( $<2$ years old). ¥Formal cytological examination is required to reliably differentiate eosinophils from other leukocytes and identify malignant cells. §HSV PCR is highly sensitive (>96\%) between days 3 and 7 of the illness, its sensitivity decreases slightly in the second week of the illness. False negatives prior to day 3 have been described. After day 10, CSF HSV IgG can be used to make a late diagnosis. TWhere available, CSF VZV IgG may be more sensitive than PCR. Testing requires the demonstration of intrathecal synthesis of VZV IgG, that is a reduced serum/CSF ratio of VZV IgG compared with the serum/CSF ratio of albumin. IICollect up to $20 \mathrm{~mL}$ blood in a clotting tube in adults and children; and up to $5 \mathrm{~mL}$ in a small child ( $<2$ years old). $\dagger+\mathrm{HIV}$ is very uncommon in children in Australia, and encephalopathy is an uncommon presentation; some experts would still undertake HIV testing as the diagnosis impacts upon possible aetiologies of encephalitis, and is treatable. $\ddagger \ddagger$ Flaviviral IgM should be tested after 5 days of symptoms. A negative result makes the diagnosis unlikely. CSF IgM is specific for these viruses and should be performed in patients in whom the diagnosis is likely in terms of risk factors, clinical and radiologic features (see Boxes 4 and 5). $\mathrm{Ag}$, antigen; CSF, cerebro-spinal fluid; CT, computed tomography scan; DWI, diffusion-weighted imaging; EEG, electro-encephalogram; FLAIR, fluid-attenuated inversion recovery; HIV, human immunodeficiency virus; HSV, herpes simplex virus; MRI, magnetic resonance imaging; PCR, polymerase chain reaction; VZV, varicella zoster virus; WBC, white blood cell.

* Table reproduced with permission from: Britton PN. Eastwood K, Paterson B, et al: Australasian Society of Infectious Diseases; Australasian College of Emergency Medicine; Australian and New Zealand Association of Neurologists; Public Health Association of Australia. Consensus guidelines for the investigation and management of encephalitis in adults and children in Australia and New Zealand (Internal Medicine Journal, Wiley Publishing Asia Pty Ltd, (C) Royal Australasian College of Physicians 2015). evidence of its yield in cohorts of patients who have encephalitis. It also introduces the various immune-mediated encephalitides, describes their clinical features and, in doing so, assists the clinician in deciding when to perform specific antibody studies. From a treatment perspective, the guideline defines optimal therapy for HSV encephalitis and outlines possible treatment strategies for other infectious and immune-mediated causes based on lower-quality evidence. In particular, it suggests when to consider empiric antimicrobial therapy and immunomodulatory therapies.

Encephalitis presents a complex challenge to clinicians. Its possibility must be suspected in a variety of presentations, and it requires the performance of a detailed clinical assessment, consultation, and judicious investigation. Unnecessary delays must be avoided, and it is essential to institute empiric therapies appropriately and provide high-quality supportive management. Optimal application of current knowledge is likely to improve diagnosis; however, even with an extensive diagnostic work-up, definitive aetiology may not be identified for $30 \%-40 \%$ of patients with encephalitis. ${ }^{7}$ Novel agents and a changing geographical distribution of known diseases are likely to be identified with improved surveillance; these possibilities should be considered where unexplained encephalitis clusters occur.

Acknowledgements: The authors of the full consensus guidelines also include: Beverley Paterson David Durrheim, Russell Dale, Allen Cheng, Chris Kenedi, James Burrow, Peter Leman, David Smith, Kerry Read and Robert Booy. Philip Britton receives funding from a National Health and Medical Research Foundation postgraduate scholarship (APP1074547), the RACP Foundation, and the Sydney Medical School and Arkhadia Fund as Dean's Fellow/Norah Theresa Hayes-Ratcliffe Lecturer.

Competing interests: No relevant disclosures.

Provenance: Not commissioned; not externally peer reviewed.

References are available online at www.mja.com.au.

1 Huppatz C, Gawarikar Y, Levi C, et al. Should there be a standardised approach to the diagnostic workup of suspected adult encephalitis? A case series from Australia. BMC Infect Dis 2010; 10: 353.

2 Kneen R, Jakka S, Mithyantha R, et al. The management of infants and children treated with aciclovir for suspected viral encephalitis. Arch Dis Child 2010; 95: 100-106.

3 Kelly C, Sohal A, Michael BD, et al. Suboptimal management of central nervous system infections in children: a multi-centre retrospective study. BMC Pediatr 2012; 12: 145.

4 Venkatesan A, Tunkel AR, Bloch KC, et al. Case definitions, diagnostic algorithms, and priorities in encephalitis: consensus statement of the International Encephalitis Consortium. Clin Infect Dis 2013; 57: 1114-1128.

5 Britton PN, Eastwood K, Paterson B, et al; Australasian Society of Infectious Diseases; Australasian College of Emergency Medicine; Australian and New Zealand Association of Neurologists; Public Health Association of Australia. Consensus guidelines for the investigation and management of encephalitis in adults and children in Australia and New Zealand. Intern Med J 2015; 45: 563-576.

6 Beaman MH, Wesselingh SL. 4: Acute community-acquired meningitis and encephalitis. Med J Aust 2002; 176: 389-396.

7 Granerod J, Ambrose HE, Davies NW, et al. Causes of encephalitis and differences in their clinical presentations in England: a multicentre, population-based prospective study. Lancet Infect Dis 2010; 10: 835-844. 